\title{
Effect of Purification, Chemical Factor and Shear Stress on Endothelial Differentiation of Human Adipose-Derived Mesenchymal Stem Cells using a Perfusion Bioreactor
}

Research Article

Gholami $\mathrm{N}^{1,3,4}$, Amoabediny $\mathrm{GH}^{2,4^{*}}$,Haghighipour $\mathrm{N}^{3^{*}}$, Hasanzadeh $\mathrm{E}^{1,3,4}$, Mohammadnejad J1 ${ }^{1}$, Shojaei $\mathrm{S}^{5}$

${ }^{1}$ Department of Life Sciences Engineering, Faculty of New Sciences \& Technologies, University of Tehran, Tehran, Iran.

${ }^{2}$ Department of Biotechnology and Pharmaceutical Engineering, Faculty of Chemical Engineering, School of Engineering, University of Tehran, Tehran, Iran.

${ }^{3}$ National Cell Bank of Iran, Pasteur Institute of Iran, Tehran, Iran.

${ }^{4}$ Research Center for New Technologies in Life Science Engineering, University of Tehran, Tehran, Iran.

${ }^{5}$ Faculty of biomedical engineering, Amirkabir University of Technology, Iran.

\section{Abstract}

The objective of this study was to answer to these principal questions that whether a more homogeneous and purified colony of Human adipose-derived mesenchymal stem cells (hASCs) has significantly higher ability of differentiation in comparison to heterogeneous hASCs colony? And also is the any synergism between chemical and mechanical motivation of hASCs?

For this purpose, CD-271+ hASCs were isolated by flow cytometry approach and their differentiation ability toward endothelial cells (ECs) was analyzed after application of mechanical shear stress and chemical growth factor. The results were compared to that of heterogeneous colony of hASCs.

Abdominal adipose tissues were isolated from previously informed and consent 25-35 year old healthy women.

A perfusion bioreactor was used for application of flow shear stress of magnitude of 4.64 dyne $/ \mathrm{cm} 2$ purified and nonpurified hASCs which lie on collagen type I coated silicon scaffold. In addition to that, cells were exposed to $50 \mathrm{ng} / \mathrm{ml}$ vascular endothelial growth factor (VEGF) for 7 days. Three endothelial specific genes (FLK-1, vWF and VE-cadherin) were selected and their expressions in RNA level were assessed by real time PCR.

Real time PCR results demonstrated that generally CD-271+ hASCs have more promising differentiation ability. Also it has been shown that highest expression of ECs specific genes is related to concurrent chemically and mechanically motivated hASCs.

In conclusion, mechanical stimulation is at least as important as chemical stimulations and a more homogeneous group of hASCs could be regarded as a more convenient source of cells for vascular tissue engineering applications.

Keywords: Adipose Tissue Stem Cells; Endothelial Cells; Purification; CD271 Marker; VEGF; Shear Stress; Perfusion Bioreactor.

Abbreviations: hASCs: Human adipose-derived mesenchymal stem cells; ECs: endothelial cells; NCBI: National Cell Bank of Iran; VEGF: vascular endothelial growth factor; FLK-1: Fetal Liver Kinase 1; vWF : von Willebrand factor; VE-cadherin: vascular endothelial cadherin; FBS: fetal bovine serum; DMEM: Dulbecco's Modified Eagle Medium; PBS: phosphate buffered saline.

\section{*Corresponding Author:}

Dr. Ghasem Amoabediny,

Department of Biotechnology and Pharmaceutical Engineering, Faculty of Chemical Engineering, School of Engineering, University of Tehran, Tehran, Iran. Tel: :+989124486374

Email: Amoabediny@ut.ac.ir

Dr. Nooshin Haghighipour,

National Cell Bank of Iran, Pasteur Institute of Iran, Tehran, Iran.

Tel: +989121404861

Email: haghighipour@pasteur.ac.ir

Received: August 02, 2016

Accepted: September 09, 2016

Published: September 12, 2016

Citation: Gholami N, Amoabediny GH, Haghighipour N, Hasanzadeh E, Mohammadnejad J, et al., (2016) Effect of Purification, Chemical Factor and Shear Stress on Endothelial Differentiation of Human Adipose-Derived Mesenchymal Stem Cells using a Perfusion Bioreactor. Int J Stem Cell Res Transplant. 04(7), $220-227$. doi: http://dx.doi.org/10.19070/2328-3548-1600034

Copyright: Amoabediny GH, Haghighipour $\mathbf{N}^{\circ}$ 2016. This is an open-access article distributed under the terms of the Creative Commons Attribution License, which permits unrestricted use, distribution and reproduction in any medium, provided the original author and source are credited. 


\section{Introduction}

Cardiovascular diseases are among the 10 leading causes of death in the world. Researchers have developed several techniques for construction of biomimicking tissue engineered blood vessels, the production of which, using endothelial or smooth muscle cells, or their mixture, requires the usage of a tubular scaffold [12].

Mesenchymal stem cells (MSCs) are undifferentiated cells which are considered as the most important cell source for regeneration of injured tissues, due to their self-renewal ability and differentiation capacity $[1,13]$. MSCs can be isolated from various tissues such as cartilage, bone marrow, adipose tissue [13]. Recently, the population of adipose-derived stem cells have been reported to be almost 8 times greater than those isolated from the bone marrow tissue [4]. Isolation of a large number of stem cells from the adipose tissue, allows us to use them without the need for their expansion. When exposed to suitable chemical factors (endothelial cell growth supplement and vascular endothelial growth factor) and shear stress in vitro, adipose-derived stem cells become morphologically and molecularly similar to endothelial cells and exhibit some major characteristics of endothelial cells such as their specific surface markers (CD31, von Willebrandfactor, endothelial nitric oxide synthase, and VE-cadherin). Therefore, adipose tissue is an available and abundant source of adult autologous stem cells which can be used in the vascular tissue engineering and regenerative medicine $[23,29]$.

Recently, CD271 has been recognized as a suitable selective marker for purification and isolation of bone marrow and adipose tissue-derived stem cells [15] . CD271-positive cells show a higher proliferation and multipotency capability, compared to the whole population of (non-purified) ASCs $[15,27]$.

It has been illustrated that mechanical stimulation of stem cells has profound effects on some the most important characteristics of these cells, such as morphology, proliferation and stem cell differentiation $[5,17]$. Endothelial cells cover the interior surfaces of blood vessels, and therefore, are of great importance in study of cardiovascular systems. In addition to chemical factors such as growth factors, endothelial cells are also affected by hemodynamic forces such as tensile stresses due to the contraction of vessel walls as well as the shear stresses produced by the frictional forces due to blood flow $[2,6]$.

In the present research, it was attempted to simulate the inherent in vivo biomechanical environment of endothelial cells. For this purpose, collagen based surface modification of tubular silicon membrane (donated by Aachen University, Germany) was accomplished. So membrane that is equipped with collagen can perform as a scaffold to provide conditions similar to the extracellular matrix for cells. Furthermore, for application of the shear forces, a designed perfusion bioreactor was used. Shear stresses were created by uniform culture medium flow in tubular collagen coated silicone membrane, according to Newton viscosity law.

According to our findings, ASCs-CD271+ is a more suitable cell source for studying the endothelial differentiation. To our best knowledge, Endothelial differentiation of CD271-based purified mesenchymal stem cells has not been previously studied. Purified and non-purified hASCs were exposed to mechanical and chemi- cal stimuli and the expression of some endothelial specific genes in two groups were compared by Real-time polymerase chain reaction (Real-time PCR) method.

\section{Materials and Methods}

\section{Cell isolation and culture}

Under approval of the Ethical Committee of National Cell Bank of Iran (NCBI), abdominal adipose tissues were isolated from 25-35 year old healthy women .Isolation of MSCs from human adipose tissue was performed according to the standard published methods [3]. with minor modifications. Minced Adipose tissue was washed with phosphate buffered saline 1X (PBS) (SigmaAldrich, St Louis, MO, USA) and centrifuged at $1400 \mathrm{rpm}$ for 5 min. Collagenase type I (Invitrogen/Thermo Fisher Scientific, Waltham, MA, USA) was added to the collected adipose tissue for enzymatic digestion ( $0.1 \mathrm{M}$ concentration) and the mixture was incubated at $37^{\circ} \mathrm{C}$ for $45 \mathrm{~min}$. Then, low-glucose Dulbecco's modified Eagle's medium (DMEM) (Gibco, USA) supplemented with 10\% fetal bovine serum (FBS) (Gibco/Thermo Fisher Scientific, Waltham, MA, USA) was added to the mixture (to deactivate collagenase function). The suspension was filtered using a $200 \mathrm{mi}-$ cron filter. The resulting suspension was centrifuged at $2000 \mathrm{rpm}$ for $5 \mathrm{~min}$. After removal of the supernatant, the remaining cell pellet was resuspended in fresh DMEM supplemented with $20 \%$ FBS and 1\% penicillin-Streptomycin and plated onto a conventional 6 or 12 -well tissue culture plate and incubated at $37^{\circ} \mathrm{C}$ with $5 \% \mathrm{CO}_{2}$. After 1 days, the culture medium exchange three times per week. After reaching $80-90 \%$ confluency, cells were passaged.

\section{Characterization of mesenchymal stem cells}

Flow Cytometry: For cell characterization, the expression of MSCs surface markers such as CD90, CD105, CD166 and CD44 as well as hematopoietic markers such as CD45 and CD34 (all antibodies from Sigma-Aldrich, USA) was studied. For this purpose, after cell counting and tripsinizing of hASCs at passage 3 , almost $5 \times 105$ cells were placed in each $15 \mathrm{ml}$ falcon and were centrifuged at $1400 \mathrm{rpm}$ for $5 \mathrm{~min}$. After removal of supernatant, deposited cells were resuspended in $100 \mu \mathrm{l}$ of PBS: FBS mixture (95\%: 5\%), followed by the addition of $2 \mu \mathrm{l}$ of each antibody and isotype to separate falcons. Then they were incubated at $4^{\circ} \mathrm{C}$ for $30 \mathrm{~min}$ followed by centrifuging at $1400 \mathrm{rpm}$ for $5 \mathrm{~min}$. After removing the supernatant and addition of $1 \mathrm{ml}$ of the mentioned substances, cell mixtures were transferred to the special flow cytometry tubes. Expression of each antibody and its isotype was examined using a BD FACS Calibur device.

Multipotential assay: In order to examine the multipotency of stem cells isolated from human adipose tissue, passage 3 cells were directed toward adipogenic and osteogenic phenotypes.

For adipogenic differentiation, cells were exposed to the adipogenic medium contained DMEM, 10\% FBS,0.5 $\mu \mathrm{M}$ IBMX (3-isobutyl-1-methylxanthine), $1 \mu \mathrm{M}$ dexamethasone, $10 \mu \mathrm{g} / \mathrm{ml}$ insulin and $100 \mu \mathrm{M}$ indomethacin (Sigma-Aldrich, USA) which was changed every 3 days. After 21 days, cells were fixed with $4 \%$ formalin for 30 min and Oil red O staining was used to examine the lipid vacuoles and adipogenic differentiation. 
For osteogenic differentiation, cells were incubated with osteogenic medium (DMEM, 10\% FBS, 100 nM dexamethasone, 10 $\mathrm{mM}$ beteglycerol phosphate and L-ascorbic acid 2-phosphate; all from Sigma-Aldrich, USA), and the medium was changed every 3 days. After 21 days, cells were fixed with paraformaldehyde for 20 min and after two times washing with PBS, Alizarin Red S staining was used to study the osteogenic cells matrix and osteogenic differentiation.

\section{Purification of hASCs population}

After characterization of hASCs, a population of isolated stem cells, at passage 2, were trypsinized and centrifuged at $1400 \mathrm{rpm}$ for 5 minutes. The remaining cell pellet was resuspended in $1 \mathrm{ml}$ of PBS. $20-30 \times 103$ cells were transferred to a $2 \mathrm{ml}$ vial and the remaining cells were transferred to another $2 \mathrm{ml}$ vial. Both vials were centrifuged at $1400 \mathrm{rpm}$ for 5 minutes and the supernatants were removed. $100 \lambda$ of isotype solution (PE mouse IgG1 isotype control; Exbio, Praha, Czech Republic) and 20-30 $\lambda$ of PBS 1X were added to the first vial and $5 \lambda$ of CD271 antibody solution (PE conjugated-mouse anti-human anti CD271; Exbio, Praha, Czech Republic) and $100 \lambda$ of PBS 1X (per 1 million cells) were added to the second vial. After incubation of both vials in refrigerator for $30 \mathrm{~min}$, they were centrifuged at $1400 \mathrm{rpm}$ for $5 \mathrm{~min}$ utes and the supernatants were removed. $100 \lambda$ of PBS $1 \mathrm{X}$ containing 10\% FBS was added to the first vial and $1 \mathrm{ml}$ of PBS $1 \mathrm{X}$ containing 10\% FBS (per 1 million cells) was added to the second vial. Cell mixtures were transferred into flow cytometry tubes and after addition of ISOTON or PBS buffer, the expression of each antibody and its isotype was examined and separation of cells expressing CD271 marker was carried out using a FACS device.

CD271 positive cells were plated onto a conventional 6-well tissue culture plate. The culture medium consisted of DMEM supplemented with $20 \%$ FBS. Cells with $80-90 \%$ confluency were passaged. Passage 3 or 4 cells were used in the experiments.

\section{Mesenchymal stem cell culture on scaffold}

A medical-grade silicone scaffold (Raumedic, Helmbrechts, Ger- many) with the inner diameter of $2 \mathrm{~mm}$ was used in this study. Hydrophobic characteristics of scaffold surface prevent cells from adhering to the surface. Therefore, in order to provide in vivo-like conditions and facilitate cell adherence to the silicone membrane, the inner surface of the scaffold was modified using collagen type I (0.1 g /100 mL PBS) (Sigma-Aldrich, USA) solution, which is a hydrophilic and biocompatible material. Briefly, after sterilization of the scaffold, collagen type I was injected into the scaffold. After tripsinizing and counting the cells, about $5 \times 105$ to $1 \times 106$ cells were injected into the collagen-coated scaffold, and then the membrane was incubated at $37^{\circ} \mathrm{C}$ and $5 \% \mathrm{CO}_{2}$ for $24 \mathrm{~h}$, in order for cells to adhere to the membrane.

\section{Endothelial differentiation using chemical stimulus}

Following cell culture on the scaffold and washing with PBS 1X, cells were incubated with endothelial differentiation medium for 7 days. The medium which was injected into the scaffold, consisted of DMEM with $3 \% \mathrm{FBS}$ and $50 \mathrm{ng} / \mathrm{mL}$ vascular endothelial growth factor (VEGF; Invitrogen, USA).The medium was change every 2 days.

\section{Endothelial differentiation using mechanical-chemical stimuli}

The scaffold is attached to the existing tube conversions at the two sides of the underlying base and then to the $40 \mathrm{ml}$-culture medium chamber, containing DMEM supplemented with $10 \%$ FBS. The device is then transferred to the incubator $\left(5 \% \mathrm{CO}_{2}\right.$ and $37^{\circ} \mathrm{C}$ ) and the peristaltic pump (RP-1; Rainin Instrument Co.) is turned on and set to the desired flow rate. Figure 1 shows the schematic view of the perfusion bioreactor.

The shear stress $(\tau)$ and flow volume $(Q)$ are calculated using Eqs. (1) and (2):

$$
\begin{array}{ll}
\tau=8 \mu(\mathrm{V} / \mathrm{D}) & \text { Eq. (1) } \\
\mathrm{V}=4 \mathrm{Q} /\left(\pi \mathrm{D}^{2}\right) & \text { Eq. (2) }
\end{array}
$$

In which $\mu$ is the viscosity $(1 \mathrm{cp}), \mathrm{V}$ is the mean velocity and $\mathrm{D}$ is

Figure 1. The schematic view of the designed perfusion bioreactor used in this study and its components. Flow of fluid passed through the collagen coated tubular scaffold with certain flow rate and created the shear stress according to Newton law of viscosity.

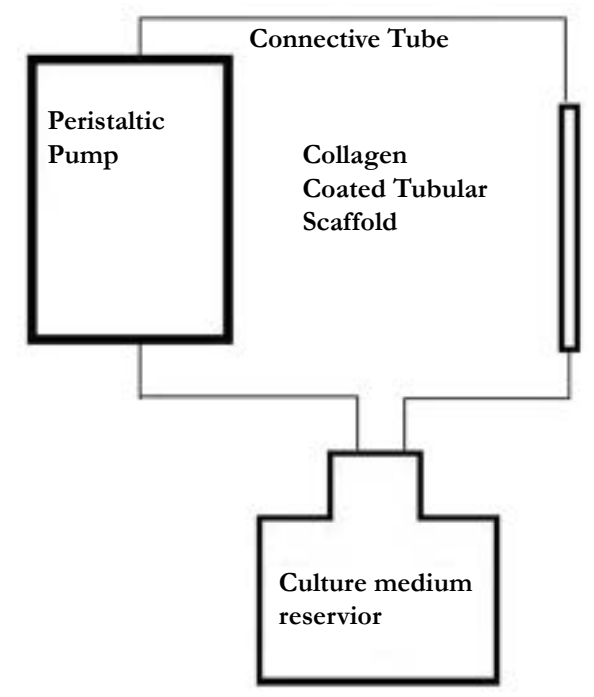


the tube diameter $(2 \mathrm{~mm})$.

The shear rate associated with the least cell detachment from the scaffold was selected since application of high stress levels causes cells to detach from the scaffold surface. Eventually the most suitable flow rate applied on mesenchymal stem cells by such perfusion bioreactor (i.e. $36 \mathrm{ml} / \mathrm{min}$ equal to 4.64 dyne $/ \mathrm{cm} 2$ for 24 hours) was chosen for mechanical-chemical induction of the cells. Then the scaffold was taken out and placed on a sterile plate. Endothelial differentiation medium was injected into the scaffold, which was then incubated at $37^{\circ} \mathrm{C}$ and $5 \% \mathrm{CO}_{2}$ for 7 days.

\section{Real-Time PCR analysis}

Endothelial differentiation of hASCs and hASCs-271+ in two test groups, namely chemical and mechanical-chemical groups, was quantitatively examined by studying the expression of three endothelial specific markers, namely vascular endothelial cadherin (VE-cadherin), von Willebrand Factor (vWF) and FLK1 (also known as KDR and VEGF receptor-2) as target genes, using Real-Time PCR technique. Glyceraldehyde 3-phosphate dehydrogenase(GAPDH) was chosen as the house keeping gene and the expression levels of target genes were normalized to GAPDH mRNA level. Total RNA was extracted using the QIAGEN RNeasy plus mini kit and cDNA synthesis was performed making use of the Quanti Tect Reverse Transcription Kit (QIAGEN, Gaithersburg, MD, USA), according to the manufacturers' instructions. Gene expression was quantified using SYBR Green Master Mix and ABI StepOne Real Time-PCR instrument (both from Applied Biosystems, Foster City, CA, and USA). Primer express software (version 3.0) was used to design the primer sequences, which are listed in Table 1.

\section{Statistical analysis}

To analyse the data, the method of comparative threshold cycle (CT) was used. The relative changes in a special target gene expression were calculated by the equation $2 \Delta \Delta \mathrm{CT}$ in which $\Delta \mathrm{CT}$ is the CT of target gene minus the CT of housekeeping gene (normalisation) and $\Delta \Delta \mathrm{CT}$ is $\Delta \mathrm{CT}$ of sample minus $\Delta \mathrm{CT}$ of Calibrator (control group).

Three independent experiments were carried out in duplicate, and data were presented as mean \pm SD. Comparisons between test groups with control group were made with t-test, a multi-factorial analysis (SPSS software). Significance was set at $\mathrm{P}<0.05$.

\section{Results}

\section{Mesenchymal characteristics of hASCs}

Flow cytometry results for hASCs population revealed the posi- tive expression of mesenchymal stem cell markers including CD90, CD105, CD166, and CD44 and the negative expression of hematopoietic markers such as CD45 and CD34 (Figure 2).

After exposing cells to adipogenic differentiation medium for 21 days and then staining with $\mathrm{Oil}$ red $\mathrm{O}$, lipid vacuoles as indications of adipogenic differentiation, could be observed. Moreover, As a result of incubating cells with osteogenic medium for 21 days, and then Alizarin Red S staining, calcium deposits, implying the occurrence of osteogenic differentiation, were also observed.

\section{Mesenchymal characteristics of CD271-based purified hADSCs}

After incubation of mesenchymal stem cells, based on CD271, the expression level of this surface marker in adipose-derived mesenchymal stem cells was analyzed using flow cytometry technique. As result of our study, ADSCs showed $1.08 \%$ of CD271 marker (Fig. 3A), which was increased to $77.8 \%$ after purification (Fig. 3B).

Results of Flow cytometry analysis of purified cell population demonstrated the positive expression of mesenchymal stem cell specific markers, namely CD90, CD105, CD166, and CD44 and the negative expression of hematopoietic markers including CD45 and CD34 (Fig. 4)

CD271 expression in passage 3 purified cells were analyzed using the flow cytometry technique, The results of which revealed that after cell culture and proliferation, $77.8 \%$ of the cells were positive for CD271 marker, which means the expression of CD271 marker by purified cell population was 72.03 times higher than that by unpurified cells (Fig. 3B).

\section{Real-time PCR results for endothelial differentiation}

mRNA levels of three endothelial specific genes, namely VEcadherin, vwf and flk-1, in both hASCs and hASCs-CD271+ cell populations were studied in chemical and mechanical-chemical groups and were compared to those for the negative control.

As it can be seen in Figure 5, in the hASCs population, the expression levels of all three genes increased compared to the negative control group and the expression level of each endothelial gene was higher in the mechanical-chemical group and it decreased in the chemical test group. In all two test groups, FLK-1 and VEcadherin showed the highest and lowest expression levels, respectively. The results of Figure 5 demonstrates that in hASCsCD271+ population, mRNA levels of all VE-cadherin, vWF and flk-1 genes were enhanced in comparison to the negative control group and the highest expression level of each gene was observed in the mechanical-chemical group. In all two test groups, vWF

Table 1. Primer sequences.

\begin{tabular}{|c|c|c|}
\hline Gene & Sequence forward & Sequence reverse \\
\hline GAPDH & 5'-ACACCCACTCCTCCACCT'T'TG-3' & 5'-TCCACCACCCTGT'TGCTGTAG-3' \\
\hline VE-cadherin & 5'-TTTCCAGCAGCCTTTCTACCAC-3' & 5'-GGAAGAACTGGCCCTTGTCAC-3' \\
\hline Flk-1 & 5'-GACTTCCTGACCTTGGAGCATCT-3' & 5'-GATTT'TAACCACGTTCTTCTCCGA-3' \\
\hline VWF & 5'-TCTGTGGATTCAGTGGATGCA-3' & 5'-CGTAGCGATCTCCAATTCCAA-3' \\
\hline
\end{tabular}


Figure 2. Flow cytometry analysis of hASCs population. hASCs were positive for CD44, CD90, CD105 and CD166, and negative for CD45 and CD34. Solid red line indicates the expression of isotype and the solid blue line indicates gene expression.

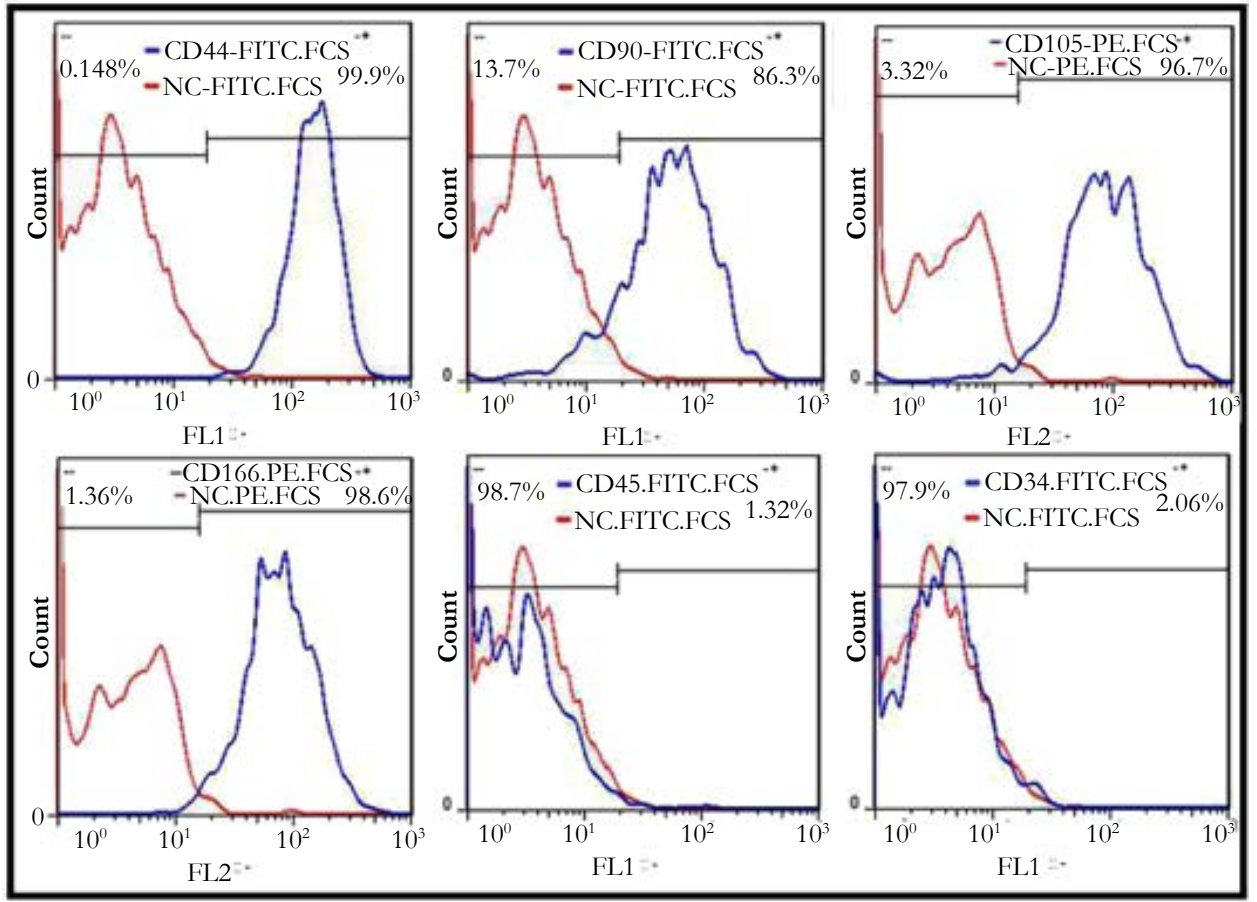

Figure 3. The expression level of CD271, before and after purification of hASCs.The expression level of CD271 in hASCs population (A) and CD271-based purified hASCs population at passage $3(\mathrm{~B})$.Solid red line indicates the expression of isotype and the solid blue line indicates gene expression.

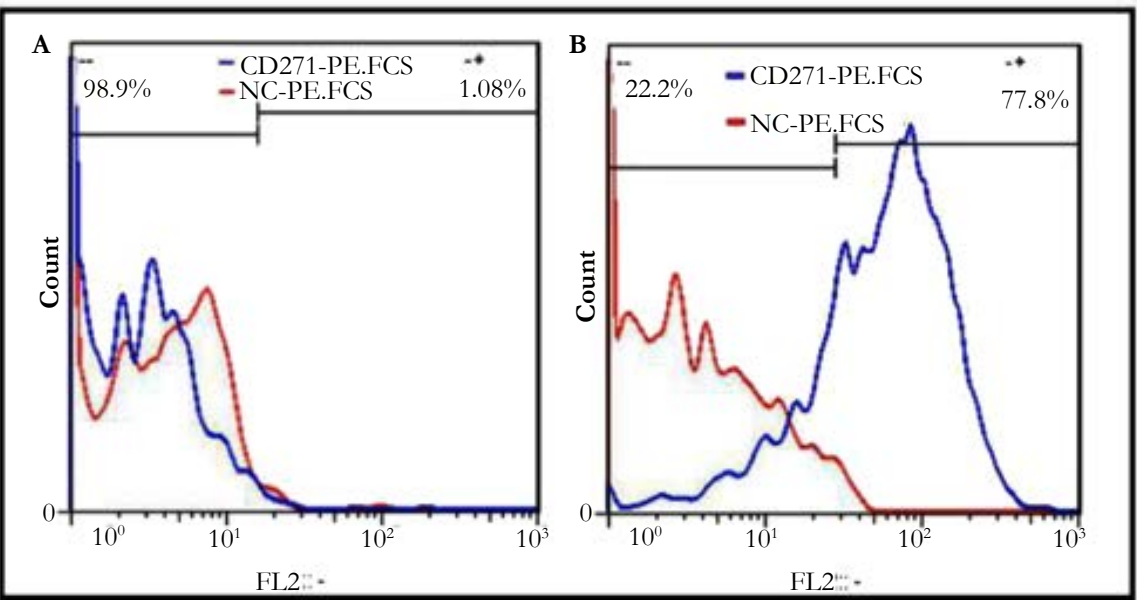

and FLK-1 showed the highest and lowest expression levels, respectively.

According to Figure 5A, FLK-1 expression level in hASCs$\mathrm{CD} 271+$ cell population in chemical and mechanical-chemical groups were 1.03 and 1.19 times lower than those of hASCs population, respectively. As it is illustrated in Figure 5B, VE-cadherin mRNA levels in hASCs-CD271+ population in mechanicalchemical and chemical groups were 1.92 and 1.28 times higher than those in the hASCs population, respectively. Figure 5C shows that the expression levels of vWF gene in hASCs-CD271+ cell population in chemical and mechanical-chemical groups were 4.01and 2.68 times higher than those in hASCs population, respectively.

\section{Discussion}

In this research, the influence of CD-271 based purification of MSCs was assessed on the differentiation ability of these cells in to endothelial cells under the effect of chemical, mechanical and mechanical-chemical stimulus.

Passage 3 cells were examined for expression of ASC specific surface markers via flow cytometry technique as well as their multipotency by studying their osteogenic and adipogenic differentiation, making use of Alizarin Red S and Oil Red O stainings, respectively. The results of these experiments confirmed the mesenchymal characteristics of isolated cells [13] .

After characterization of adipose-derived mesenchymal stem 
Figure. 4. Flow cytometry analysis of hASCs-CD271+ population. hASCs-CD271+ population were positive for CD44, CD90, CD105 and CD166, and negative for CD5 and CD34. Solid red line indicates the expression of isotype and the solid blue line indicates gene expression.

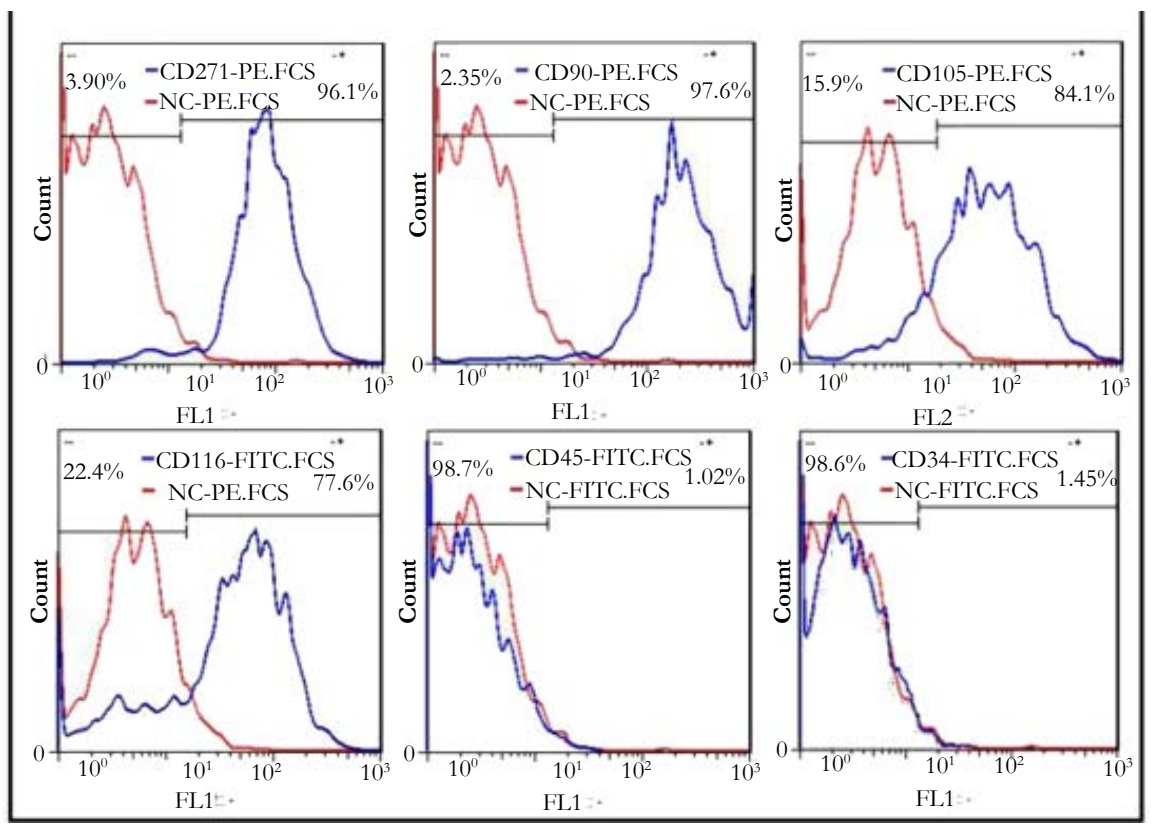

Figure 5. Relative mRNA expression levels of FLK-1(A), VE-cadherin (B) and vWF (C) in different test groups in hASCs and hASCs-CD271+.Error bars indicate standard deviation. Paired t-test was performed to compare gene expression between each test group and negative control. Parameter *describes significant difference $(\mathrm{p}<0.05)$.

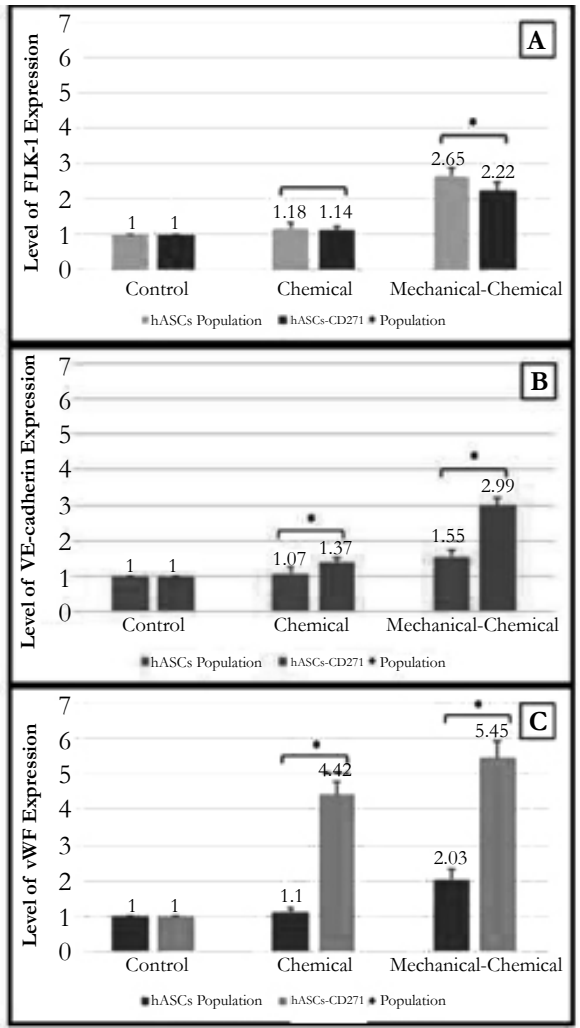

cells, a number of MSCs were purified based on CD271 marker by FACS technique. The results of characterization revealed that both purified and unpurified cell populations were positive for mesenchymal stem cell markers, namely CD90, CD105, CD166, and CD44 and negative for hematopoietic markers including CD45 and CD34, which proved the mesenchymal nature of the two cell populations. As it was expected from previous reports, the isolated adipose-derived MSCs are a heterogeneous popula- tion of cells in which those MSCs bearing CD271 marker, have a higher differentiation ability, compared to those heterogeneous stem cells isolated from adipose tissues $[8,16]$.

Previous researches suggest that special concentration of VEGF during a specific time period can enhance endothelial differentiation of MSCs significantly [14] . 
In addition to chemical factors, mechanical stimulation can be regarded as an important contributor of differentiation process. Shear stress is an important stimulus which enhances the acquisition of some of endothelial cells vital characteristics in ASCs. It plays vital roles in physiological responses of blood vessels during EC signaling and may affect the expression of growth factors [24, 26]. The physiologic range of shear stress in the body is 5-25 dyn/ $\mathrm{cm} 2$, and in most relevant studies, in order to direct stem cells differentiation toward endothelial cells, such magnitudes of shear stress have been applied for $24 \mathrm{~h}[11,29]$.

While some previous studies demonstrate that shear stress alone is not an endothelial inducing factor by itself and it is necessary for it to be accompanied by chemical growth factors $[24,26]$, some other researches imply that shear stress even solely is able to induce endothelial differentiation [19, 20, 21]. Not only shear forces allow the regulation of growth factor expression in cells [29] but also it has been reported that VEGF and shear stress have synergistic effects on balancing the signaling and gene expression in endothelial cells [22, 25]. Therefore, in this paper, VEGF alone or a combination of the shear stress of $4.64 \mathrm{dyn} /$ $\mathrm{cm} 2$ produced by a perfusion bioreactor and VEGF were used to induce endothelial differentiation on purifiedand and unpurified cell populations. The effect of purification can be best analyzed by comparing the results of two groups.

The three specific genes of endothelial cells, namely Flk-1, vWF and VE-cadherin play major roles in angiogenesis process and endothelium maturation [14]. The expression of VEGFR2 (Flk1), vWF and VE-cadherin has been studied in a time-dependent manner from the early stages of differentiation of stem cells toward vascular endothelial cells [9]. The expression of VEGFR2, as an early marker for endothelial lineage development has been observed in the first 3-4 days of differentiation, while CD31, vWF and VE-cadherin have been recognized as the markers of mature endothelial cells [10].

As it was expected, after performing each of chemical or mechanical-chemical tests on purified and unpurified cell populations, the expression levels of all endothelial markers were increased, compared to those in the negative control group [19, 20, 21, 25].

In chemical test group, the expression levels of Flk-1, VE-cadherin and vWF were increased by 1.18, 1.07 and 1.1 times in unpurified cell population, and by 1.14, 1.37 and 4.42 times in the purified cell population, respectively, compared to the negative control group. This finding is in consistence with the results of other studies on the role of VEGf factor in endothelial differentiation $[8,14,29]$. VEGF by its interaction with cell receptors of FLT-1 (VEGFR-1) and FLK-1 (VEGFR-2) acts an important role in vascular differentiation and by activating Flk-1-PI3K-AktHDAC3-p53-p21 pathway cause to a sound increase in express of particular endothelium markers i.e FLK-1, CD31, vWF and VE-cadherin $[18,28]$.

In the chemical test group, the expression levels of Flk-1, VEcadherin and $\mathrm{vWF}$ in purified cell population were $1.03 \%$ lower and $1.28 \%$ and $4.01 \%$ higher than those in unpurified cell population. Since Flk-1 in one hand and VE-cadherin and vWF on the other hand are among the early and late endothelial markers respectively and vWF is a characteristic marker of mature endothe- lial cells, the elevation of these genes demonstrates an improvement in endothelial differentiation $[9,10,14,25]$.

In comparison to the negative control group, in the mechanicalchemical group, the expression levels of Flk-1, VE-cadherin and vWF were increased by $2.65,1.55$ and 2.03 times in unpurified, and by 2.22, 2.99 and 5.45 times in purified cells, respectively; that is in support of all recent achivements related to the positive effect of applying a combination of VEGF and shear stress in endothelial differentiation of mesenshymal stem cells [19, 22, 25]. The higher gene expression in the mechanical-chemical group compared to the other test group can be explained by the synergistic effect of shear stress and endothelial growth factor on endothelial gene expression in the MSCs populations [19, 22, 25] . It has been shown that shear stress and VEGF has an analogous signaling pathway in up regulation of endothelial differentiation of MSCs [10].

The considerably higher mRNA levels of vWF and VE-cadherin in the purified cell population imply the positive synergetic influence of the combination of chemical and mechanical signals on these two endothelial markers in the purified cells. As mentioned earlier, this indicates the improvement in endothelial differentiation toward the final stages of differentiation as a result of purification of mesenchymal stem cells (hASCs-CD271+) in the mechanical-chemical test group.

To our best knowledge, In this study, endothelial differentiation of CD271-based purified mesenchymal stem cells was examined for the first time. This purified cell population showed a $72.03 \%$ increase in CD271 marker expression compared to the unpurified population of adipose-derived mesenchymal stem cells.

Former researches demonstrated that CD-271 based purified MSCs have higher chondrogenic, adipogenic and osteogenic differentiation ability $[8,16]$. Our findings proved that CD-271+ population of MSCs have higher potential for differentiation into endothelial cells in comparison to unpurified inhomogeneous population of MSCs. It was found that the purification of the MSCs population and application of shear stress and exposure to VEGF have profound effects on elevation of early and late expression of endothelial specific genes in MSCs. Beside that the influence of purification was well illustrated by comparing the results of purified and unpurified tests groups.

The findings of this paper could be helpful in the future applications of vascular tissue engineering.

\section{Acknowledgment}

The author would like to thank Dr. Shokrgozar and Mrs. Salehinik and Mrs. Ghazizadeh for their scientific assistance.

\section{References}

[1]. Amin S, Banijamali SE, Tafazoli-Shadpour M, Shokrgozar MA, Dehghan MM, Haghighipour N, et al., (2014) Comparing the effect of equiaxial cyclic mechanical stimulation on GATA4 expression in adipose-derived and bone marrow-derived mesenchymal stem cells. Cell biology int. 38(2): 21927

[2]. Ando J, Yamamoto K (2013) Flow detection and calcium signalling in vascular endothelial cells. Cardiovascu Res. 99(2): 260-8.

[3]. Bunnell BA, Flaat M, Gagliardi C, Patel B, Ripoll C (2008) Adipose-derived 
stem cells: isolation, expansion and differentiation. Methods. 45(2): 115-20.

[4]. Cuevas-Diaz Duran R, González-Garza MT, Cardenas-Lopez A, ChavezCastilla L, Cruz-Vega DE, Moreno-Cuevas JE (2013) Age-Related Yield of Adipose-Derived Stem Cells Bearing the Low-Affinity Nerve Growth Factor Receptor. Stem cells int. 2013: 372164

[5]. Haghighipour N, Heidarian S, Shokrgozar MA, Amirizadeh N (2012) Differential effects of cyclic uniaxial stretch on human mesenchymal stem cell into skeletal muscle cell. Cell biology int. 36(7): 669-75.

[6]. Haghighipour N, Tafazzoli-Shadpour M, Shokrgozar MA, Amini S (2010) Effects of cyclic stretch waveform on endothelial cell morphology using fractal analysis. Artif organs. 34(6): 481-90.

[7]. Huang NF, Li S (2008) Mesenchymal stem cells for vascular regeneration. Regen Med. 3(6): 877-92.

[8]. Kuçi S, Kuçi Z, Kreyenberg H, Deak E, Pütsch K, Huenecke S, et al., (2010) CD271 antigen defines a subset of multipotent stromal cells with immunosuppressive and lymphohematopoietic engraftment-promoting properties. Haematologica. 95(4): 651-9.

[9]. Levenberg S, Golub JS, Amit M, Itskovitz-Eldor J, Langer R (2002) Endothelial cells derived from human embryonic stem cells. Proc Natl Acad Sci U S A. 99(7): 4391-6.

[10]. Luo Z, Wang G, Wang W, Xiao Q, Xu Q (2010) Signalling pathways that regulate endothelial differentiation from stem cells. Front Biosci (Landmark Ed). 16: 472-85

[11]. Nagatomi J (2011) Mechanobiology Handbook. CRC Press.

[12]. Nemeno-Guanzon JG, Lee S, Berg JR, Jo YH, Yeo JE, Nam BM, et al., (2012) Trends in tissue engineering for blood vessels. J BioMed Biotechnol. 2012: 956345.

[13]. Nombela-Arrieta C, Ritz J, Silberstein LE (2011) The elusive nature and function of mesenchymal stem cells. Nat Rev Mol Cell Biol. 12(2): 126-31.

[14]. Oswald J, Boxberger S, Jørgensen B, Feldmann S, Ehninger G, Bornhäuser $\mathrm{M}$, et al., (2004) Mesenchymal stem cells can be differentiated into endothelial cells in vitro. Stem cells. 22(3): 377-84.

[15]. Quirici N, Scavullo C, de Girolamo L, Lopa S, Arrigoni E, Deliliers GL, et al., (2009) Anti-L-NGFR and-CD34 monoclonal antibodies identify multipotent mesenchymal stem cells in human adipose tissue. Stem Cells Dev. 19(6): 915-25.

[16]. Quirici N, Soligo D, Bossolasco P, Servida F, Lumini C, Deliliers GL (2002) Isolation of bone marrow mesenchymal stem cells by anti-nerve growth factor receptor antibodies. Experimental Hematol. 30(7): 783-91.

[17]. Salehi-Nik N, Amoabediny G, Pouran B, Tabesh H, Shokrgozar MA, Haghighipour N, et al., (2013) Engineering parameters in bioreactor's design: a critical aspect in tissue engineering. BioMed Research International.
2013:15.

[18]. Scioli MG, Bielli A, Gentile P, Mazzaglia D, Cervelli V, Orlandi A (2014) The Biomolecular Basis of Adipogenic Differentiation of Adipose-Derived Stem Cells. Int J Mol Sci. 15(4): 6517-26.

[19]. Shoajei S, Tafazzoli-Shahdpour M, Shokrgozar MA, Haghighipour N (2014) Alteration of human umbilical vein endothelial cell gene expression in different biomechanical environments. Cell Bio Int. 38(5): 577-81.

[20]. Shojaei S, Farokhi M, Omidvar R, Mottaghitalab F, Haghighipour N, Shokrgozar M, et al., (2013) Essential Functionality of Endometrial and Adipose Stem Cells in Normal and Mechanically Motivated Conditions. Journal of Biomaterials and Tissue Engineering. 3(5): 581-588.

[21]. Shojaei S, Tafazzoli-Shadpour M, Shokrgozar MA, Haghighipour N, Safaei N, Mahdian R et al., ( 2013) The Influence of Cyclic and Uniform Shear Stresses Concurrent with Cyclic Stretch on the Gene Expression of Human Umbilical Vein Endothelial Cells. Journal of Biomaterials and Tissue Engineering. 3(6): 673-8.

[22]. Shojaei S, Tafazzoli-Shahdpour M, Shokrgozar MA, Haghighipour N (2013) Effects of mechanical and chemical stimuli on differentiation of human adipose-derived stem cells into endothelial cells. Int J Artif Organs. 36(9): 663-73.

[23]. Vater C, Kasten P, Stiehler M (2011) Culture media for the differentiation of mesenchymal stromal cells. Acta Biomater. 7(2): 463-77.

[24]. Wang H, Riha GM, Yan S, Li M, Chai H, Yang H, et al., (2005) Shear stres induces endothelial differentiation from a murine embryonic mesenchymal progenitor cell line. Arterioscler Thromb Vasc Bio. 25(9): 1817-23.

[25]. Wu CC, Chao YC, Chen CN, Chien S, Chen YC, Chien CC, et al., (2008) Synergism of biochemical and mechanical stimuli in the differentiation of human placenta-derived multipotent cells into endothelial cells. J Biomech. 41(4): 813-21.

[26]. Yamamoto K, Takahashi T, Asahara T, Ohura N, Sokabe T, Kamiya A, et al., (2003) Proliferation, differentiation, and tube formation by endothelial progenitor cells in response to shear stress. J Appl Physiol. 95(5): 2081-8.

[27]. Yamamoto N, Akamatsu H, Hasegawa S, Yamada T, Nakata S, Ohkuma M, et al., (2007) Isolation of multipotent stem cells from mouse adipose tissue. Journal of dermatological science. 48(1): 43-52.

[28]. Zeng L, Xiao Q, Margariti A, Zhang Z, Zampetaki A, Patel S, et al., (2006) HDAC3 is crucial in shear-and VEGF-induced stem cell differentiation toward endothelial cells. J Cell Biol. 174(7): 1059-69.

[29]. Zhang P, Moudgill N, Hager E, Tarola N, DiMatteo C, McIlhenny S, et al., (2010) Endothelial differentiation of adipose-derived stem cells from elderly patients with cardiovascular disease. Stem Cells Dev. 20(6): 977-88. 